\title{
A MODIFIED ZING IODIDE-OSMIUM TETROXIDE STAINING METHOD FOR NERVE TERMINALS IN SPINAL GORDS OF THE RAT
}

\author{
Yasuhiko IBATA, * Yoshiaki NOJYO, Tadao MATSUURA \\ and Junzo OCHI** \\ Department of Anatomy, Kyoto Prefectural University \\ of Medicine, Kyoto, Japan
}

Received for publication May 30, 1972

\begin{abstract}
The synaptic terminals of the ventral horn of rat spinal cords were demonstrated by electron microscopy using a modification of the zinc iodideosmium tetroxide staining technique in which zinc iodide was substituted for a mixture of zinc powder and iodine sublimate.

The best staining reaction consistent with tissue preservation was obtained by incubating specimens for 16 hours at $4^{\circ} \mathrm{G}$ in solutions containing $1 \%$ or $2 \%$ zinc iodide. Synaptic vesicles staining black were observed in both $F$ and $S$ types of synaptic terminals. With the exception of some parts of the myelin sheath no other neuronal or glial elements gave a positive reaction.

The modified technique was compared with that of other investigators and the possible nature of the reaction between the stain and the synaptic vesicles was briefly discussed.
\end{abstract}

A zinc iodide-osmium tetroxide (ZIO) staining technique comparable to the silver impregnation method of demonstrating nerve terminals by optical microscopy was described by Maillet in 1962 (8) and Jabonero in 1964 (6). It was subsequently adapted to electron microscopic studies of nerve terminals in the subfornical organ and neuromuscular junctions (1), spinal cord (7), superior cervical ganglion (10), pineal body $(11,12)$ mammalian olfactory bulb (5), goldfish spinal cord (4) and cephalopod brain (9). The stain has an affinity for synaptic vesicles in nerve terminals and some authors have claimed that it could be used to demonstrate cholinergic nerve terminals $(1,9)$ but this has been disputed by others $(10,11,12)$. The precise specificity of the procedure is still in doubt (2).

All those $(1,4,5,7,9,11)$ who have so far used the $\mathrm{ZIO}$ reaction to demonstrate nerve terminals by electron microscopy employed a mixture of zinc powder and iodine bisublimate in the form of a suspension. This was well shaken and filtered before mixing with osmium tetroxide solution. In the present study we used cheaper and more easily prepared zinc iodide which does not require filtration.

\footnotetext{
* Reprint request should be sent to: Dr. Y. Ibata, Department of Anatomy, Kyoto Prefectural University of Medicine, Kawaramachi-Hirokoji, Kyoto, Japan.

**Present Address: Department of Anatomy and Embryology, Tokyo Metropolitan Institute for Neurosciences, Fuchu City, Tokyo Japan.
} 


\section{MATERIALS AND METHODS}

Twenty albino rats lightly anaesthetised with nembutal were perfused with paraformaldehyde fixative adjusted to $\mathrm{pH} 7.4$ with $0.05 \mathrm{M}$ phosphate buffer. After perfusion blocks of spinal cord $1 \mathrm{~mm}$ thick were removed from the cervical and lumbar enlargements and divided into two batches. One was further fixed for two hours at $4^{\circ} \mathrm{C}$ in $2.5 \%$ glutaraldehyde, the other in $6.25 \%$ glutaraldehyde, the $\mathrm{pH}$ being maintained at 7.4 with $0.1 \mathrm{M}$ phosphate buffer. Samples from each batch were then incubated at $4^{\circ} \mathrm{C}$ for periods of 8,16 or 20 hours in a modified ZIO mixture.

MODIFIED ZIO MIXTURE

Solution $A$

$3.3 \%$ or $6.6 \%$ zinc iodide solution

3 parts

(Solution was facilitated by adding four drops of IN $\mathrm{HCl}$ )

Solution $B$

Tris-HCl buffer

4 parts

Solution $C$

$4 \%$ osmium tetroxide solution

3 parts

The final mixture contains either $1 \%$ or $2 \%$ zinc iodide, is yellow brown to dark brown in color, and has a $\mathrm{pH}$ of about 5.0.

After incubation the blocks of tissue were dehydrated in a graded series of acetone and embedded in Epon 812. Semi-thin sections of the ventral horn of the cords were stained and examined before proceeding to thin sectioning. Thin sections were cut on a Porter Blum II Ultramicrotome, stained with uranyl acetate and lead acetate and examined in a JEM 7 electron microscope. A parallel series of sections impregnated with ZIO by the method of Kawana et al. (7) was also prepared for purposes of comparison.

\section{RESULTS}

The effects on nerve terminals in the ventral horns of the cords produced by varying the final concentration of zinc iodide and the duration of incubation were as follows:

1. $1 \%$ zinc iodide; 8 hours incubation.

A very weak positive reaction was observed in the presynaptic terminals of the cord. The terminal shown in Fig. 1 contained many agranular synaptic vesicles, some large dense cored vesicles, mitochondria and a few intensely stained structures which we consider to be impregnated synaptic vesicles. The ZIO reaction was minimal in agranular synaptic vesicles and absent in pre-and postsynaptic membranes and mitochondria.

2. $1 \%$ zinc iodide ; 16 hours incubation.

A more marked reaction was observed in the nerve terminals in this case.

The number of densely stained structures believed to be synaptic vesicles was 


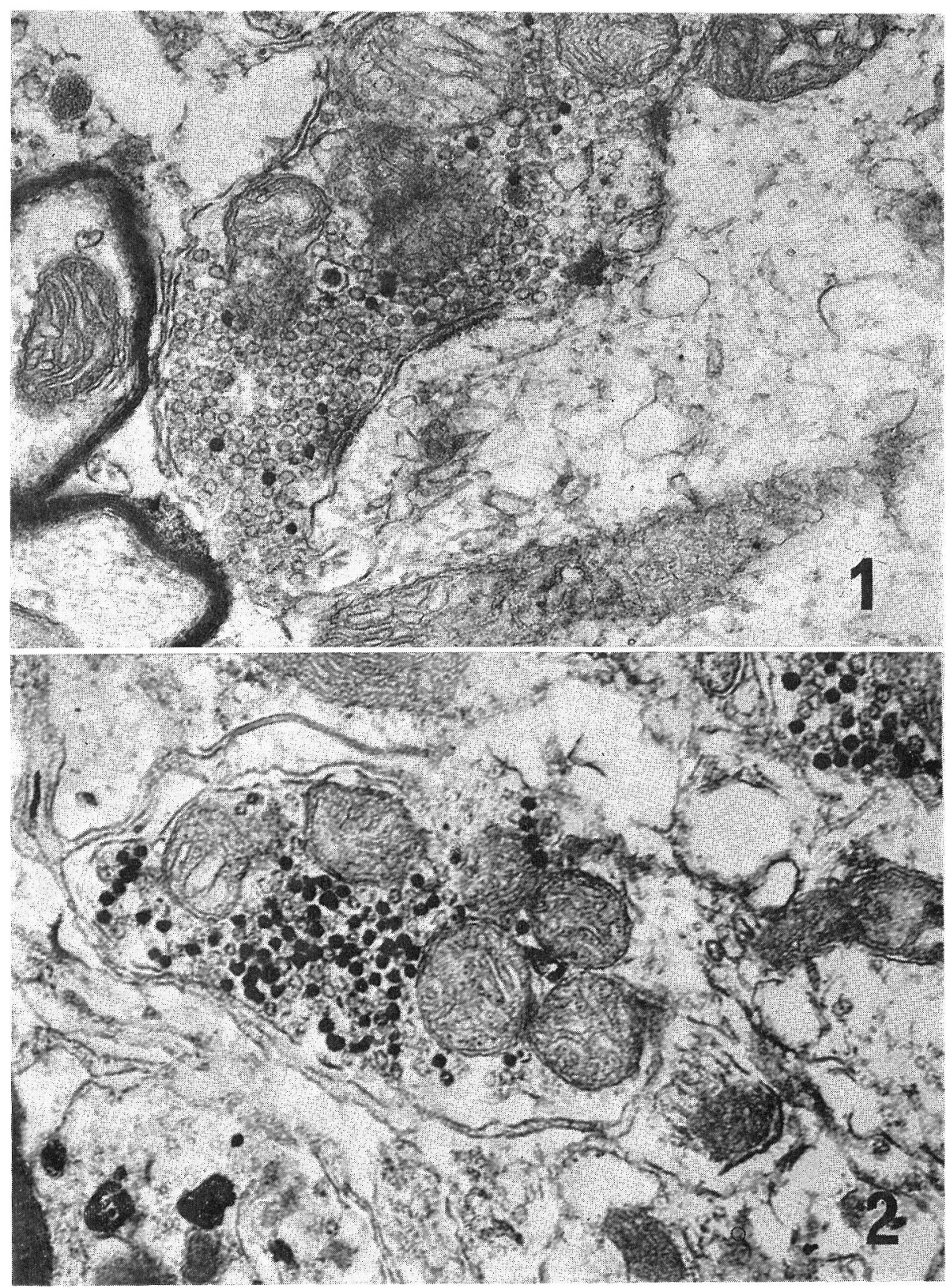

FIG. 1. 1\% zinc iodide, 8 hours incubation.

Only a few of the synaptic vesicles (agranular vesicles) contained in the presynaptic terminal have taken up the stain. $\times 36,000$.

FIG. 2. $1 \%$ zinc iodide, 16 hours incubation.

Black staining particles representing synaptic vesicles are considerably increased in number. $\times 40,000$. 


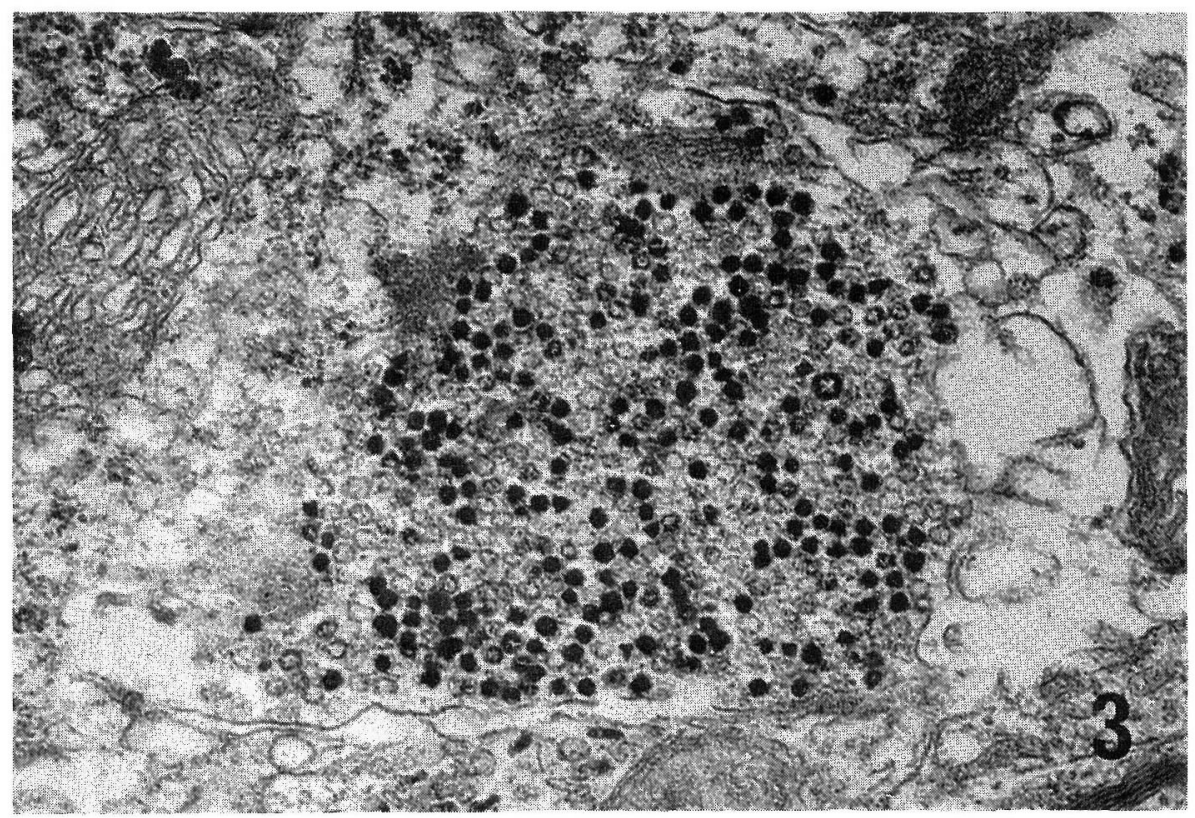

FIG. 3. $1 \%$ zinc iodide, 24 hours incubation.

Nearly half of the synaptic vesicles are heavily stained. $\times 37,000$.

greatly increased (Fig. 2). Synaptic membranes and mitochondria were not stained.

3. $1 \%$ zinc iodide $; 20$ hours incubation.

Numerous synaptic vesicles gave a positive reaction, probably to a greater extent than when incubated for 16 hours (Fig. 3). Nevertheless the number of positive vesicles was less than half the total number in the terminals. Once again there was no observable response in synaptic membranes or mitochondria. Two types of terminal are found in the spinal cord, type $S$ containes a preponderance of spherical vesicles, type $F$ a preponderance of flattened vesicles $(3,13)$. In this study both types of terminal showed a positive $\mathrm{ZIO}$ reaction, the only difference being in the shape of the vesicle.

4. $2 \%$ zinc iodide; 8 hours incubation.

The results appeared to be intermediate between those obtained by incubating for 8 hours and 16 hours in $1 \%$ zinc iodide. Some dark staining vesicles were observed in the terminals but the majority were unaffected (Fig. 4).

5. $2 \%$ zinc iodide; 16 hours incubation.

Under these conditions the ZIO reaction was much more apparent than in the 8 hour preparations, but the total number of stained vesicles was less than $50 \%$, a comparable proportion to that obtained in the $1 \%$ zinc iodide/20 hour incubation experiment (Fig. 5). No other elemental structure gave a positive reaction except for myelin sheath, in which the innermost lamellae were deeply stained (Fig. 6). 


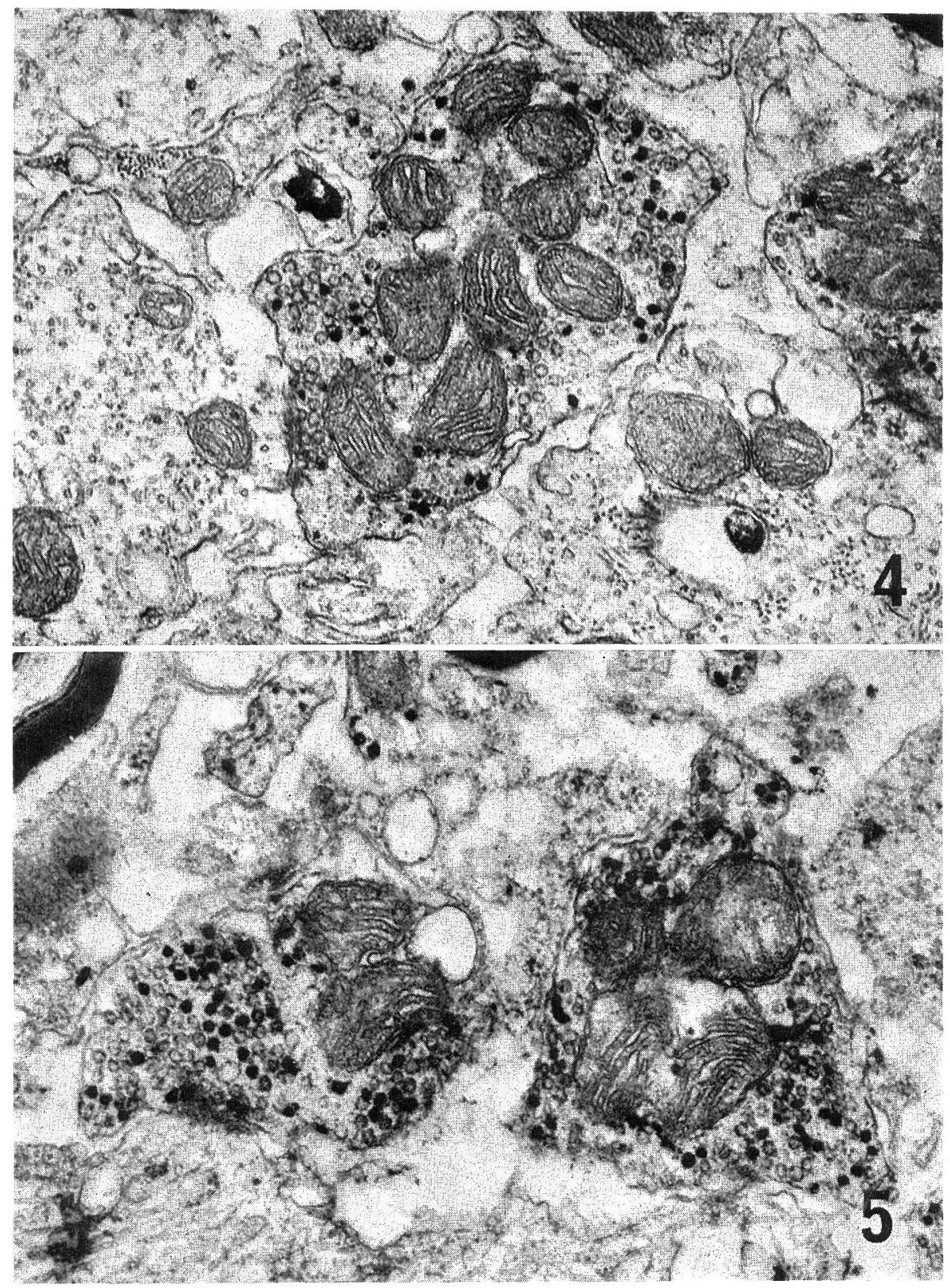

FIG. 4. $2 \%$ zinc iodide, 8 hours incubation.

Relatively few vesicles have stained. $\times 30,000$.

FIG. 5. 2\% zinc iodide, 16 hours incubation.

Two synaptic terminals are shown, both containing a high proportion of stained vesicles. $\times 32,000$. 

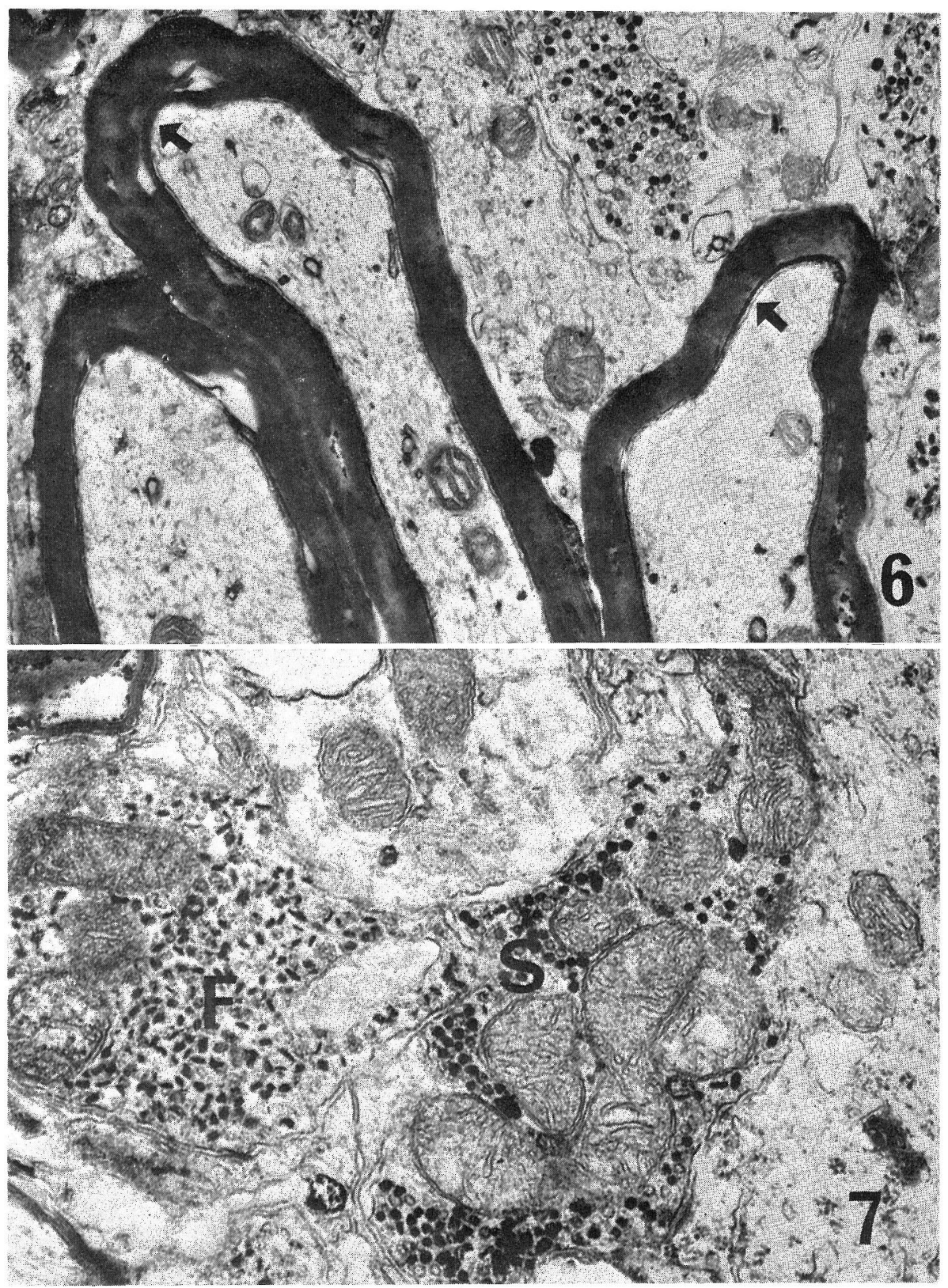

FIG. 6. The innermost lamellae of the myelin sheath are heavily stained (arrows) in addition to the vesicles. $2 \%$ zinc iodide, 16 hours incubation. $\times 33,000$.

Frg. 7. Spherical (S) and flattened (F) synaptic vesicles showing a positive ZIO reaction. $2 \%$ zinc iodide, 20 hours incubation. $\times 30,000$. 
6. $2 \%$ zinc iodide ; 20 hours incubation.

Almost $50 \%$ of the vesicles in both $S$ and $F$ terminals were heavily stained, the remainder showed no reaction (Fig. 7). Even after this long incubation period the synaptic membranes, mitochondria and other neuronal and glial elements, apart from myelin sheath lamellae, failed to stain.

In order to assess the possible effect on the ZIO reaction of varying the strength of the glutaraldehyde prefixation solution we performed all the above experiments in parallel, one series being prefixed in $2.5 \%$ glutaraldehyde, the other in $6.25 \%$ glutaraldehyde. The reaction observed in the synaptic vesicles appeared to be identical in both cases. Although we regard glutaraldehyde fixation as an essential step in this technique its concentration appears to be unimportant.

We also found that washing the specimens with Tris-HCl buffer was a prerequisite of the ZIO reaction since non specific precipitation occurred after incubation in specimens that were not washed in this way.

The degree of the ZIO reaction on synaptic vesicles for each of the six incubation conditions was divided into 5 categories as shown in Table 1 .

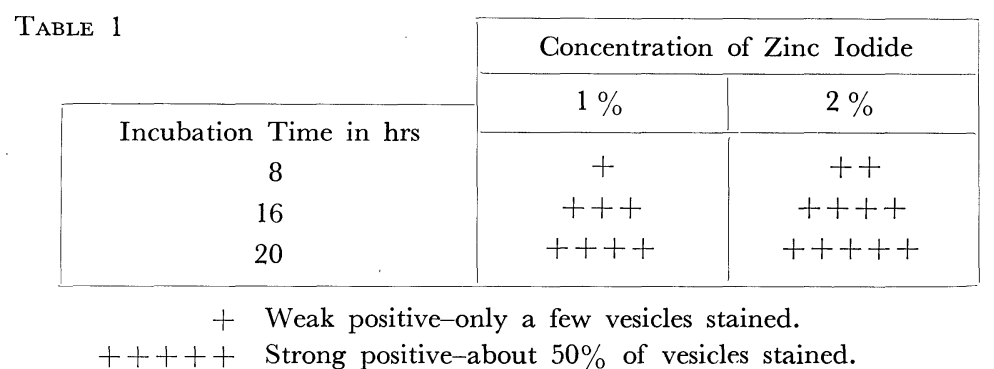

\section{DISCUSSION}

Reproducible results were obtained by using a modified ZIO method of demonstrating synaptic terminals in the ventral horn of the spinal cord of the rat. The method offers advantages of economy and ease of preparation of the reaction medium, without sacrificing the reaction specificity and tissue preservation described by other investigators.

We found the optimum concentration of zinc iodide for staining synaptic vesicles to be $1 \%$ or $2 \%$, which accords with the final concentration in the filtered solutions used by other workers $(1,4,5,7,9,11)$. Adjustment of $\mathrm{pH}$ of the incubation medium does not seem to be critical as similar results were obtained with our method using pH 5.0 and that of Kawana et al. using $\mathrm{pH}$ 6.5. This does not necessarily apply to other structures e.g. neuronal and glial elements. Our results indicate a positive ZIO reaction in some lamellae of myelin sheaths, while others have described heavy staining of the Golgi complex and lysosomes of nerves and glial cells $(2,9)$.

In our method there seemed to be a close parallel between the degree of the staining reaction of synaptic vesicles on one hand, and the duration of incubation and concentration of zinc iodide on the other. 
Non specific precipitation of osmium encountered by Martin et al. (9) did not occur in our preparations even after incubation times as long as 20 hours. We concluded that a 16 hour incubation period in a zinc iodide-osmium tetroxide mixture containing $1 \%$ or $2 \%$ zinc iodide was the optimum staining condition consistent with tissue preservation. Using this method all the synaptic terminals of the ventral horn of the spinal cord gave a positive ZIO reaction. This result agrees with that of Kawana et al. (7).

Almost $50 \%$ of the synaptic vesicles in those specimens subjected to long periods of incubation were found to be ZIO positive. All the vesicles, whether stained or not, were identical in shape and size, and a reason for the difference in the staining reaction must be sought. Three possible explanations for the failure of some vesicles to take up stain are that the vesicular contents lack affinity for ZIO, that the vesicular membrane is impervious to ZIO solution, and that the quantity of vesicular contents is insufficient for the reaction to occur. If the first assumption is accepted, it suggests the possibility of the existence of more than one transmitter in a single ending. There is no morphological evidence to support this theory, all vesicles having the same shape, size and density, but there may be a difference in the chemical nature of the vesicular contents. This is probably composed of neurotransmitter and an associated carrier substance, protein in nature. It has not been shown which of these substances is ZIO positive. Uchizono (13) postulated that in the ventral horn of the cat spinal cord the $S$ type terminals, containing spherical vesicles only, were excitatory, while $F$ type terminals containing a preponderance of flattened vesicles were inhibitory. If spherical and flattened vesicles contain different transmitters the ZIO reaction may involve carrier substance, which is common to both vesicles, rather than transmitter.

The possibility of different transmitters reacting simultaneously with ZIO must also be taken into account. Support for this may be found in the publications of the following authors:-

Pellegrino de Iraldi and Subero (12) demonstrated the ZIO reaction in serotonin stored in vesicles of rat pineal nerve endings, which could be prevented by specifically inhibiting serotonin with DL-p-Chlorophenylalanine. Matus (10) showed that chromaffin cells of the superior cervical ganglion known to contain catecholamine were ZIO positive. Akert and Sandri (1) working with axon terminals in the subfornical organ and myoneural junctions suggested that the ZIO reaction occurred specifically at cholinergic nerve endings, and Martin et al. (9) using cephalopod nerves reached a similar conclusion.

It is clear from consideration of all the evidence that the specificity of the ZIO reaction cannot be precisely defined. Nevertheless, it provides a valuable method of demonstrating and classifying certain synaptic nerve endings.

\section{REFERENCES}

1. Akert, K. and Sandri, C.: An electron microscopic study of zinc iodide-osmium impregnation of neurons. I. Staining of synaptic vesicles at cholinergic junctions. Brain Res., 7; 286, 1968.

2. Akert, K., Moor, H., Pfenninger, K. and Sandri, C.: Contribution of new impregnation methods and freeze etching to the problems of synaptic fine structure. In Progress in Brain Research, edited by K. Akert and P. G. Waser. Elsevier, Amsterdam, vol. 31, p. 223, 1969. 
3. Bodian, D.: An electron microscopic characterization of classes of synaptic vesicles by means of controlled aldehyde fixation. J. Cell Biol., 44; 115, 1970.

4. Dennison, M. E.: Zinc iodide-osmium tetroxide reaction in the spinal cord of goldfish. Brain Res., 27; 357, 1971a.

5. Dennison, M. E.: Electron stereoscopy as a means of classifying synaptic vesicles. J. Cell Sci., $8 ; 525,1971 \mathrm{~b}$.

6. Jabonero, V., Über die Brauchbarkeit der Osmiumtetroxyd-Zinkjodid-Methode zur Analyse der vegetativen Peripherie. Acta neuroveg. (Wien), 26; 184, 1964.

7. Kawana, E., Akert, K. and Sandri, G.: Zinc iodied-osmium tetroxide impregnation of nerve terminals in the spinal cord. Brain Res., 16; 325, 1969.

8. Maillet, M.: La technique de Champy àl' osmium ioduré de potassium et la modification de Maillet àl' osmiumi-oduré de zinc. Trab. Inst. Cajal Invest. biol., 54; 1, 1962.

9. Martin, R., Barlow, J. and Miralto, A.: Application of the Zinc iodide-osmium tetroxide impregnation of synaptic vesicles in cephalopod nerves. Brain Res., 15; 1, 1969.

10. Matus, A. I.: L ltrastructure of the superior cervical ganglion fixed with zinc iodide and osmium tetroxide. Brain Res., 17; 195, 1970.

11. Pellegrino de Iraldi, A. and Gueudet, R.: Action of reserpine on the osmium tetroxide zinc iodide reactive site of synaptic vesicles in the pineal nerves of the rat. Z. Zellforsch., 91; 178, 1968.

12. Pellegrino de Iraldi, A. and Suburo, A. M.: Action of p-chlorophenylalanine on the synaptic vesicles from rat pineal nerves. Experientia, 15; 289, 1971.

13. Uchizono, K.: Exitatory and inhibitory synapses in the cat spinal cord. Jap. J. Physiol., $16 ; 570,1966$. 